REVISTA INTERNACIONAL DE CIENCIAS DEL DEPORTE International Journal of Sport Science

Rev. int. cienc. deporte

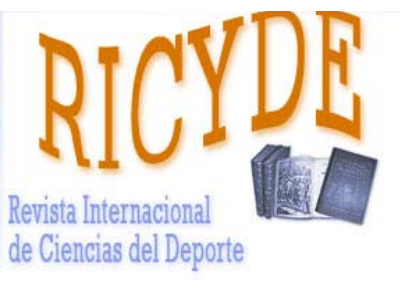

International Journal of Sport Science VOLUMEN IX - AÑO IX

Páginas:333-341 ISSN:1885-3137

No 34 - Octubre - 2013

\title{
Aplicación de la dinamometría isocinética para establecer perfiles de riesgo de lesión isquiosural en futbolistas profesionales \\ The use of isokinetic dynamometry to establish risk profiles of hamstring injury in professional football players
}

\author{
Víctor Moreno-Pérez, David Barbado-Murillo, Casto Juan-Recio \\ Universidad Miguel Hernández de Elche \\ César M. Quesada-de-la-Gala \\ Servicios médicos Elche C.F \\ Francisco J. Vera-García \\ Universidad Miguel Hernández de Elche
}

\section{Resumen}

Las lesiones en la musculatura isquiosural son frecuentes en el fútbol profesional y han sido relacionadas con el desequilibrio entre la fuerza de los flexores y extensores de rodilla. El objetivo de nuestro estudio fue relacionar los valores de fuerza máxima excéntrica de flexores/concéntrica de extensores (Flex exc30/Ext $_{\text {con240 }}$ ) y el ángulo de máximo momento de fuerza con la incidencia de lesiones de isquiosurales sufridas por los jugadores a lo largo de una temporada, con el fin de proporcionar valores de referencia de este parámetro que permitan la prevención de este tipo de lesiones. Veinte jugadores de un equipo de la Segunda División de la Liga de Fútbol Profesional realizaron bilateralmente un test isocinético de flexión excéntrica de rodilla a $30 \%$ s y extensión concéntrica de rodilla a $240 \%$ s durante la pretemporada. Tras calcular la ratio $\mathrm{Flex}_{\text {exc30/Ext }}$ con240 y el ángulo de máximo momento de fuerza, se registraron las lesiones sufridas por los jugadores a lo largo de la temporada. Los resultados mostraron que dos de los cinco jugadores lesionados presentaron ratios Flex exc30/Ext $_{\text {con240 }}$ menores de 0,89, valor que ha sido utilizado previamente para determinar el desequilibrio entre la musculatura flexora y extensora de rodilla y el riesgo de lesión (Croisier, Ganteaume, Binet, Genty, y Ferret, 2008). Además, otros dos de los jugadores lesionados obtuvieron ratios entre 0,93 y 1,00. Por otro lado, no se encontraron diferencias en el ángulo de máximo momento de fuerza entre jugadores lesionados y no lesionados. Estos resultados indican que la ratio Flex $_{\text {exc30/ }} / \mathrm{Ext}_{\mathrm{con} 240}$ puede ser un índice útil para determinar el riesgo de lesión isquiosural en futbolistas profesionales.

Palabras clave: fútbol; lesión de isquiosurales; desequilibrio de fuerza; prevención.

\section{Abstract}

Hamstring injuries are common in professional football and have been related to the imbalance between knee flexor and extensor strength. The aim of our study was to relate the maximum strength values of flexor eccentric/extensor concentric $\left(\right.$ Flex $_{\text {exc30/Ext }}$ En240 $_{2}$ ) and peak torque angle with the hamstring injuries incidence sustained by football players during a season to provide reference values of this parameter to allow injuries prevention. Twenty players of a Second Division team of the Football Professional League bilaterally performed an isokinetic test of eccentric knee flexion at $30 \% / \mathrm{s}$ and con-

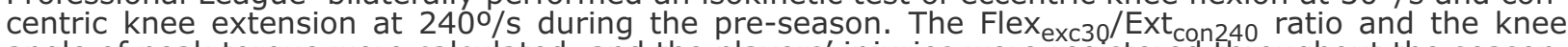
angle of peak torque were calculated, and the players' injuries were registered throughout the season. The results showed that two of the five injured players had Flex exc30/Ext $_{\text {con240 }}$ ratios lower than 0,89, value that has been previously used to determine the imbalance between knee flexor and extensor strength and the risk of injury (Croisier, Ganteaume, Binet, Genty, and Ferret, 2008). In addition, another two injured players obtained ratios between 0,93 and 1,00. On the other hand, no differences in the knee angle of peak torque between injured and non-injured players were found. These results indicate that the Flex exc30/Ext $_{\text {con240 }}$ ratio may be a useful index to determine the risk of hamstring injury in professional football players.

Key words: football; hamstring injury; strength imbalance; prevention.

Correspondencia/correspondence: Francisco J. Vera-García

Centro Investigación del Deporte de la Universidad Miguel Hernández de Elche, España

Email: fvera@umh.es 
Moreno-Pérez, V.; Barbado-Murillo, D.; Juan-Recio, C.; Quesada-de-la-Gala, C.M.; Vera-García, F.J. (2013). Aplicación de la dinamometría isocinética para establecer perfiles de riesgo de lesión isquiosural en futbolistas profesionales. RICYDE. Rev. int. cienc. deporte. 34(9), 333-341. http://dx.doi.org/10.5232/ricyde2013.03403

\section{Introducción}

$\mathrm{D}$ ebido a las altas exigencias neuromusculares y articulares de la práctica del fútbol, existe una importante incidencia de lesión en este deporte: 10-35 lesiones por cada 1000 horas jugadas (Dvorak y Junge, 2000). Esto supone un problema importante para el fútbol profesional, ya que las lesiones de los futbolistas tienen una gran repercusión sobre los intereses deportivos y económicos de los clubes (Small, McNaughton, Greig, y Lovell, 2009). Entre las lesiones más comunes en el fútbol profesional destacan las distensiones y/o roturas de los músculos isquiosurales, que representan el $37 \%$ del total de lesiones de los futbolistas (Ekstrand, Hägglund, y Waldén, 2011). Además, destaca como aspecto importante, su elevada tasa de reincidencia (Petersen y Hölmich, 2005), entre el 12-16\% de los casos (Ekstrand, Hägglund, y Waldén, 2011; Woods, Hawkins, Maltby, Hulse, Thomas, Hodson, 2004).

Existen varios factores de riesgo relacionados con la elevada incidencia de sufrir lesión muscular en los isquiosurales. De ellos destacan la edad del jugador (Gabbe, Bennell, y Finch, 2006), haber sufrido previamente esta lesión (Orchard, 2001), la fatiga muscular (Greig y Siegler, 2009; Small y col., 2009), el déficit de flexibilidad (Witvrouw, Danneels, Asselman, D'Have, y Cambier, 2003), una pobre estabilidad lumbo-pélvica (Sherry y Best, 2004) y desequilibrios de fuerza entre la musculatura extensora y flexora de la rodilla (Croisier, 2004; Croisier, Forthomme, Namurois, Vanderthommen, y Crielaard, 2002; Jönhagen, Németh, y Eriksson, 1994; Yeung, Suen, Yeung, 2009). Este último factor es de gran importancia, ya que la lesión de los isquiosurales ocurre comúnmente en acciones balísticas de extensión de rodilla, como el sprint o el chut, cuando los músculos flexores se activan excéntricamente para frenar rápidamente el movimiento generado previamente por los músculos extensores de rodilla (Bennell, Wajswelner, Lew, Schall-Riaucour, Leslie, Plant, y Cirone, 1998; Petersen y Hölmich, 2005). Por ello, el desequilibrio entre la fuerza en contracción excéntrica de la musculatura flexora de la rodilla y la fuerza en contracción concéntrica de la musculatura extensora de la rodilla ha sido utilizado como índice para predecir la aparición de lesión isquiosural en deportistas (Croisier y col., 2002).

Croisier y col. (2002) realizaron diversos protocolos con dinamometría isocinética con objeto de encontrar un test que relacionase desequilibrios de fuerza entre grupos musculares y el riesgo de sufrir lesión isquiosural. En concreto utilizaron una ratio mixta de fuerza excéntrica de flexores/concéntrica de extensores que se mostró como un indicador válido de riesgo de lesiones. Esta ratio la desarrollaron combinando dos velocidades extremadamente diferentes, es decir, $30 \%$ s en la contracción excéntrica de flexores de rodilla y $240 \%$ s en la contracción concéntrica de extensores de rodilla $\left(\mathrm{Flex}_{\mathrm{exc} 30} / \mathrm{Ext}_{\mathrm{con} 240}\right)$, con objeto de aproximarse a las condiciones biomecánicas que se producen durante las distintas acciones que requieren de extensiones balísticas de rodilla (Croisier y col., 2002), como las anteriormente citadas sprint y el chut. Atendiendo a esta ratio, inicialmente Croisier y col. (2002) determinaron que valores menores de 0,80 indican un alto riesgo de sufrir lesión isquiosural, si bien un estudio posterior de este grupo de investigación situó la ratio $\mathrm{Flex}_{\mathrm{exc} 30} / \mathrm{Ext}_{\mathrm{con} 240}$ en 0,89 (Croisier y col., 2008).

Otro de los parámetros obtenidos a partir de las pruebas isocinéticas que ha sido relacionado con el riesgo de lesión isquiosural es el ángulo donde la musculatura flexora de rodilla alcanza su máximo momento de fuerza concéntrica (Brockett, Morgan, y Proske, 2004; Proske, Morgan, Brockett, y Percival, 2004). Se ha observado que este ángulo es menor (mayor flexión de rodilla) en atletas que han sufrido lesión previa y que, por tanto, están catalogados como atletas con alto riesgo de lesión isquiosural (Brockett y col., 2004). 
Moreno-Pérez, V.; Barbado-Murillo, D.; J uan-Recio, C.; Quesada-de-la-Gala, C.M.; Vera-García, F.J. (2013). Aplicación de la dinamometría isocinética para establecer perfiles de riesgo de lesión isquiosural en futbolistas profesionales. RICYDE. Rev. int. cienc. deporte. 34(9), 333-341. http://dx.doi.org/10.5232/ricyde2013.03403

El objetivo del estudio fue relacionar los valores de ratio $\mathrm{Flex}_{\mathrm{exc} 30} / \mathrm{Ext}_{\mathrm{con} 240} \mathrm{y}$ el ángulo de máximo momento de fuerza con la incidencia de lesiones de isquiosurales sufridas por los jugadores a lo largo de una temporada, con el fin de proporcionar valores de referencia de este parámetro que permitan la prevención de este tipo de lesiones.

\section{Método}

\section{Participantes}

En el estudio participaron 20 jugadores de fútbol $(27,07 \pm 3,56$ años; 75,65 \pm 4,69 kg; 1,78 \pm $0,05 \mathrm{~m}$ ) pertenecientes a un equipo de la Segunda División de la Liga de Fútbol Profesional (Liga Adelante). De acuerdo con el equipo médico del club, ninguno de los jugadores estaba lesionado en el momento de la ejecución del test. Además, se utilizó un cuestionario ad hoc para conocer el historial de lesiones musculares sufridas por los jugadores. La pierna dominante de cada futbolista se determinó según su preferencia para el golpeo de balón. Todos los participantes firmaron un consentimiento informado antes del test. El estudio se llevó a cabo de acuerdo al Código de Ética de la Asociación Médica Mundial (Declaración de Helsinki, 1975) y su procedimiento fue aprobado por el comité ético de la Universidad Miguel Hernández de Elche antes de comenzar el estudio.

\section{Procedimiento}

Debido a las características de la muestra, la dificultad en cuanto a disponibilidad de la misma y siguiendo la metodología de estudios previos con muestras similares (Lehance, Binet, Bury, y Croisier, 2009), cada uno de los participantes realizó el test en una única sesión en el mes de julio de 2011, durante la pretemporada de la Liga 2011/2012. Durante la temporada ninguno de los jugadores realizó un trabajo específico preventivo para evitar posibles lesiones en la musculatura isquiosural. La prueba se realizó con el dinamómetro isocinético BIODEX System 4 (Biodex Medical Systems, New York, USA) y consistió en una evaluación isocinética bilateral de la musculatura extensora y flexora de la rodilla. El protocolo incluyó esfuerzos concéntricos de los músculos extensores de la rodilla, que consistieron en tres series de tres repeticiones máximas a una velocidad angular de $240^{\circ} / \mathrm{s}$, y esfuerzos excéntricos de los músculos flexores de la rodilla, que consistieron en tres series de tres repeticiones máximas a una velocidad angular de $30 \%$. Los jugadores fueron animados continuamente durante la ejecución de la prueba con objeto de garantizar que el esfuerzo fuera máximo.

Todas las medidas fueron precedidas por un calentamiento estandarizado de $10 \mathrm{~min}: 5 \mathrm{~min}$ de pedaleo en el nivel tres $(75-80 \mathrm{~W})$ de una bicicleta estática Technogym Bike Med (Technogym Trading S.A., Barcelona, España) y 5 min de ejercicios estáticos de flexibilidad de la musculatura implicada. Para realizar el test se colocó al deportista sentado en el dinamómetro con un ángulo de flexión coxo-femoral de $105^{\circ}$ (figura 1). Posteriormente, se alineó el eje de rotación articular de la rodilla (determinado mediante la palpación de la cara externa del cóndilo lateral femoral con flexión a $90^{\circ}$ ) con el eje del dinamómetro y el eje longitudinal de la pierna, con la palanca del dinamómetro. El punto de aplicación de la fuerza (zona almohadillada de empuje y tracción) se fijó en el tercio distal de la pierna, inmediatamente por encima del maléolo tibial. Para estandarizar el test y evitar que cada jugador pudiera utilizar una técnica de ejecución diferente, se inmovilizó al sujeto con correas situadas en el tórax, la pelvis y el muslo (figura 1). Se fijó en $90^{\circ}$ el rango de movimiento de la rodilla, tomando como valor de inicio la máxima extensión activa de la articulación. Asimismo, con objeto de familiarizar al deportista con el uso del dinamómetro, antes de iniciar el test se realizaron cinco repeticiones submáximas. Durante las mediciones, el 
Moreno-Pérez, V.; Barbado-Murillo, D.; J uan-Recio, C.; Quesada-de-la-Gala, C.M.; Vera-García, F.J. (2013). Aplicación de la dinamometría isocinética para establecer perfiles de riesgo de lesión isquiosural en futbolistas profesionales. RICYDE. Rev. int. cienc. deporte. 34(9), 333-341. http://dx.doi.org/10.5232/ricyde2013.03403

momento de la fuerza peso de la palanca del dinamómetro y de la extremidad inferior fue calculado y compensado automáticamente por el dinamómetro.

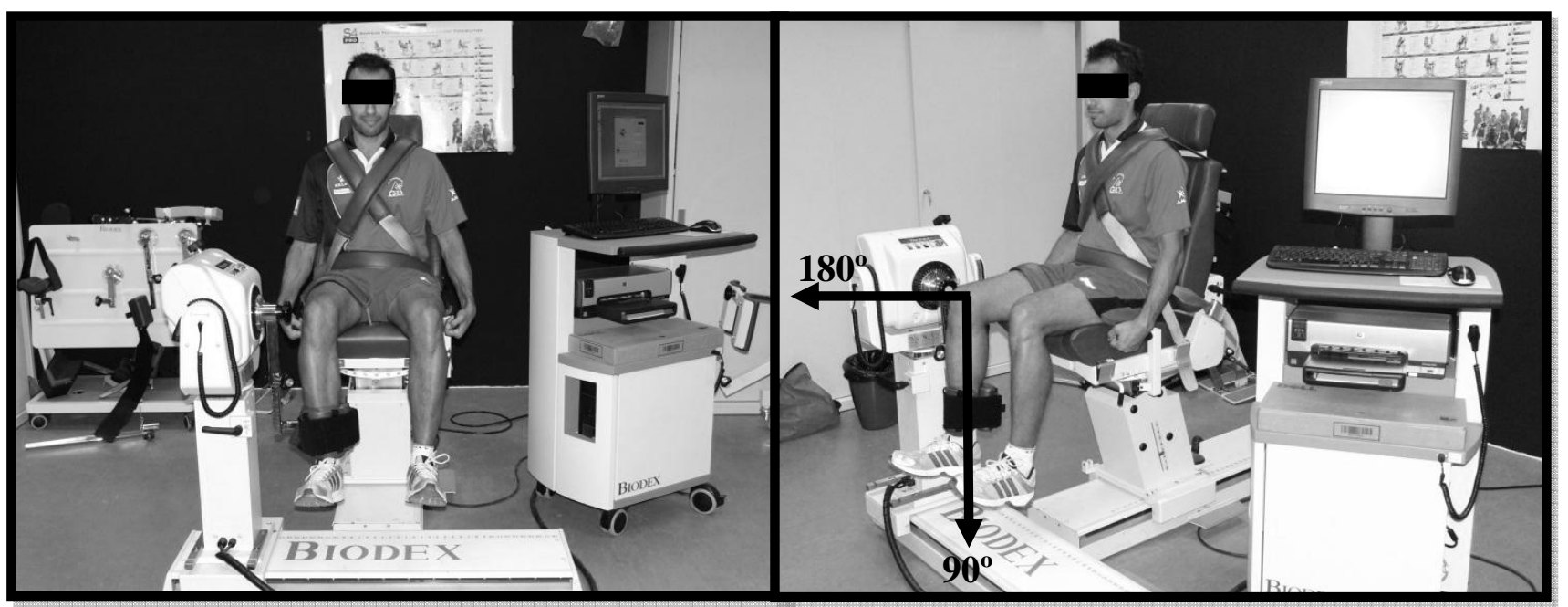

Figura 1. Imágenes de un deportista antes de comenzar el test. En la imagen de la derecha se ha representado el rango del movimiento articular.

\section{Tratamiento de los datos}

Los parámetros utilizados para valorar el rendimiento del deportista en el test fueron el momento de fuerza máximo $(\mathrm{N} \cdot \mathrm{m})$ y el ángulo $\left(^{\circ}\right)$ al que se consiguió dicho momento. Ambos parámetros se obtuvieron del software proporcionado por el dinamómetro isocinético. Se

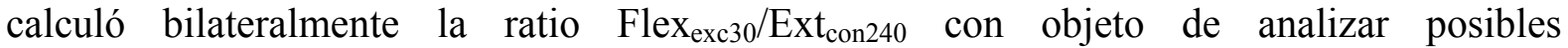
descompensaciones entre los músculos antagonistas. Partiendo de los trabajos de Croisier y col. (2002, 2008), se determinó la existencia de descompensación muscular y riesgo de lesión cuando se obtuvieran ratios $\mathrm{Flex}_{\mathrm{exc} 30} / \mathrm{Ext}_{\mathrm{con} 240}$ inferiores a 0,89 .

Para la comparación de las variables de fuerza analizadas entre jugadores lesionados y no lesionados se utilizó la prueba no paramétrica U de Mann-Whitney para muestras independientes. Para ver la relación entre los valores de las ratios obtenidas por cada uno de los jugadores y el hecho de sufrir o no una lesión, se realizó un análisis de correlación biserial puntual $\left(\mathrm{r}_{\mathrm{bp}}\right)$. La hipótesis nula fue rechazada al nivel de significación del 95\% $(\mathrm{p} \leq 0,05)$. El análisis estadístico de los datos se realizó con el programa SPSS 18.0 (SPSS Inc., Chicago, IL, USA).

Para determinar las lesiones que el deportista sufrió durante la temporada 2011/2012 se realizó un seguimiento por parte de los servicios médicos del club, que registraron las lesiones sufridas por cada futbolista, el grupo muscular afectado y la gravedad de la lesión. Para establecer la gravedad de la lesión se utilizó la clasificación de la Asociación Española de Médicos de Equipos de Fútbol (AEMEF), que se basa en el tiempo de baja de los futbolistas: leve (1-7 días), moderada (8-28 días) y grave (más de 28 días).

\section{Resultados}

En la tabla 1 se presentan los resultados obtenidos en el test isocinético por los cinco jugadores del equipo que presentaron lesiones deportivas en los isquiosurales a lo largo de la temporada 2011/2012 (25\% del total de la muestra). Todas estas lesiones se produjeron en la pierna no dominante. Por otra parte, el $80 \%$ de las lesiones se produjeron durante la primera vuelta del campeonato. De los cinco jugadores referidos, dos presentaron ratios Flex $_{\mathrm{exc} 30} /$ Ext $_{\text {con }}$ menores de 0,89 y otros dos, ratios comprendidas entre 0,89 y 1,00. De los 15 
Moreno-Pérez, V.; Barbado-Murillo, D.; J uan-Recio, C.; Quesada-de-la-Gala, C.M.; Vera-García, F.J. (2013). Aplicación de la dinamometría isocinética para establecer perfiles de riesgo de lesión isquiosural en futbolistas profesionales. RICYDE. Rev. int. cienc. deporte. 34(9), 333-341. http://dx.doi.org/10.5232/ricyde2013.03403

jugadores que no sufrieron lesión, únicamente tres tuvieron ratios Flex $_{\text {exc } 30} /$ Ext $_{\text {con }}$ entre $0,90 \mathrm{y}$ 0,97 (los otros 12 tuvieron valores mayores que 1,00).

Tabla 1. Resultados del test isocinético de flexión/extensión de rodilla de la pierna no dominante en los jugadores lesionados.

\begin{tabular}{|c|c|c|c|c|c|c|c|c|}
\hline Jugador & $\begin{array}{c}\text { M } \\
\text { Ext }_{\text {con240 }} \\
(\mathbf{N} \cdot \mathbf{m})\end{array}$ & $\begin{array}{c}\text { M } \\
\text { Flex }_{\text {exc30 }} \\
(\mathbf{N} \cdot \mathbf{m})\end{array}$ & $\begin{array}{c}\text { A } \\
\text { Ext }_{\text {con240 }} \\
\left(^{\circ}\right)\end{array}$ & $\begin{array}{c}\text { A } \\
\text { Flex } \\
\left(_{\text {exc30 }}{ }^{\circ}\right)\end{array}$ & Ratio & $\begin{array}{c}\text { Grupo } \\
\text { Muscular } \\
\text { Lesionado }\end{array}$ & $\begin{array}{c}\text { Gravedad } \\
\text { de la } \\
\text { Lesión }\end{array}$ & $\begin{array}{l}\text { Momento } \\
\text { de la lesión }\end{array}$ \\
\hline 1 & 167,5 & 217,1 & 100 & 170 & 1,17 & $\mathrm{BF}(1)$ & Moderada & $\begin{array}{c}\text { 28-10-2011 } \\
\text { (jornada 11) }\end{array}$ \\
\hline 2 & 140,9 & 120 & 95 & 170 & 0,85 & $\mathrm{BF}(1)$ & Grave & $\begin{array}{c}\text { 11-2-2012 } \\
\text { (jornada 24) }\end{array}$ \\
\hline 3 & 142,9 & 132,3 & 114 & 169 & 0,93 & ST (1) & Moderada & $\begin{array}{c}\text { 3-12-2011 } \\
\text { (jornada 16) }\end{array}$ \\
\hline 4 & 229 & 229,3 & 89 & 162 & 1,00 & $\begin{array}{c}\mathrm{BF}(2) ; \mathrm{ST} \\
\text { (1) }\end{array}$ & Moderada & $\begin{array}{l}\text { 17-9-2011 } \\
\text { (jornada 5) }\end{array}$ \\
\hline 5 & 151,1 & 107,4 & 104 & 160 & 0,71 & $\mathrm{BF}(1)$ & Leve & $\begin{array}{l}\text { 27-11-2011 } \\
\text { (jornada 15) }\end{array}$ \\
\hline
\end{tabular}

Variables: máximo momento de fuerzas (M) y ángulo del máximo momento de fuerzas (A) de los extensores de rodilla en una contracción isocinética concéntrica a $240^{\circ} / \mathrm{s}\left(\right.$ Ext $\left._{\text {con240 }}\right)$ y de los flexores de rodilla en una contracción excéntrica a $30 \%$ s $\left(\mathrm{Flex}_{\mathrm{exc30}}\right)$; Ratio del máximo momento de fuerzas entre flexores en una contracción isocinética concéntrica a $240 \%$ y extensores en una contracción excéntrica a $30 \%$ s (Ratio).

Grupos Musculares: BF = biceps femoral; $\mathrm{ST}=$ Semitendinoso. Entre paréntesis se indica el número de lesiones durante la temporada.

Gravedad de la lesión: Leve (1-7 días); Moderada (8-28 días); Grave (>28 días).

En la tabla 2 se muestra la comparación entre los parámetros obtenidos en el test isocinético para cada uno de los grupos (lesionados y no lesionados). Los jugadores lesionados presentaron una ratio Flex $_{\mathrm{exc} 30} / \mathrm{Ext}_{\mathrm{con}}$ en la pierna no dominante (la única en la que se observó lesión) significativamente menor que los jugadores no lesionados.

No se encontraron diferencias en los ángulos donde se alcanzó el pico del momento de fuerza de la musculatura isquiosural entre los jugadores lesionados y no lesionados. Tampoco se encontraron en el ángulo entre la pierna dominante y no dominante de los jugadores lesionados.

El análisis de correlación biserial puntual entre los valores obtenidos y el hecho de sufrir o no una lesión, mostró una correlación negativa buena $\left(\mathrm{r}_{\mathrm{bp}}=-0,614 ; \mathrm{p}=0,004\right)$ cuando se tuvieron en cuenta las ratios obtenidas en la pierna no dominante y una correlación negativa moderada-baja $\left(\mathrm{r}_{\mathrm{bp}}=-0,371 ; \mathrm{p}=0,018\right)$ cuando se tuvieron en cuenta las ratios obtenidas en ambas piernas. 
Moreno-Pérez, V.; Barbado-Murillo, D.; Juan-Recio, C.; Quesada-de-la-Gala, C.M.; Vera-García, F.J. (2013). Aplicación de la dinamometría isocinética para establecer perfiles de riesgo de lesión isquiosural en futbolistas profesionales. RICYDE. Rev. int. cienc. deporte. 34(9), 333-341. http://dx.doi.org/10.5232/ricyde2013.03403

Tabla 2. Comparación de las variables obtenidas del test isocinético de flexión/extensión de rodilla entre lesionados y no lesionados. Media (desviación típica)

\begin{tabular}{|c|c|c|c|c|c|}
\hline & PIERNA & $\begin{array}{l}\text { TODOS } \\
(\mathrm{N}=20)\end{array}$ & $\begin{array}{l}\text { LESIONADOS } \\
\quad(\mathrm{N}=5)\end{array}$ & $\begin{array}{l}\text { NO LESIONADOS } \\
\qquad(\mathrm{N}=15)\end{array}$ & $\begin{array}{c}\text { LESIONADOS } \\
\text { vs. } \\
\text { NO LESIONADOS } \\
\text { Sig. }\end{array}$ \\
\hline \multirow{2}{*}{$M-E_{x t}{ }_{\text {con240 }}(\mathrm{N} \cdot \mathrm{m})$} & PD & $156,21(23,73)$ & $162,60(17,19)$ & $154,06(25,68)$ & 0,445 \\
\hline & PND & $155,22(29,93)$ & $166,32(36,60)$ & $151,53(27,84)$ & 0,553 \\
\hline \multirow{2}{*}{ M-Flex ${ }_{\mathrm{exc} 30}(\mathrm{~N} \cdot \mathrm{m})$} & PD & $189,78(34,84)$ & $183,02(31,83)$ & $193,37(37,11)$ & 0,445 \\
\hline & PND & $185,58(41,53)$ & $157,20(53,07)$ & $193,70(33,42)$ & 0,197 \\
\hline \multirow{2}{*}{ A-Ext ${ }_{\text {con240 }}\left({ }^{\circ}\right)$} & PD & $109,95(17,45)$ & $103,60(8,29)$ & $112,53(18,93)$ & 0,266 \\
\hline & PND & $106,85(9,63)$ & $99,00(10,02)$ & $109,00(8,98$ & 0,119 \\
\hline \multirow{2}{*}{ A-Flex $\operatorname{exc30}\left({ }^{\circ}\right)$} & $\overline{P D}$ & $162,15(7,60)$ & $164,40(8,38)$ & $161,40(7,47)$ & 0,395 \\
\hline & PND & $163,25(5,79)$ & $166,20(4,81)$ & $162,27(5,90)$ & 0,230 \\
\hline \multirow{2}{*}{$\underset{\text { Flex }_{\text {exc30 }} / \text { Ext }_{\text {con240 }}}{\text { Ratio }}$} & PD & $1,22(0,20)$ & $1,12(0,12)$ & $1,26(0,21)$ & 0,066 \\
\hline & PND & $1,21(0,28)$ & $0,93(0,17)$ & $1,30(0,25)$ & $0,015^{*}$ \\
\hline
\end{tabular}

Variables: máximo momento de fuerzas (M) y ángulo del máximo momento de fuerzas (A) de los extensores de rodilla en una contracción isocinética concéntrica a $240 \% \mathrm{~s}\left(\right.$ Ext $\left._{\text {con240 }}\right)$ y de los flexores de rodilla en una contracción excéntrica a $30 \%\left(\right.$ Flex $\left._{\text {exc30 }}\right)$; Ratio del máximo momento de fuerzas entre

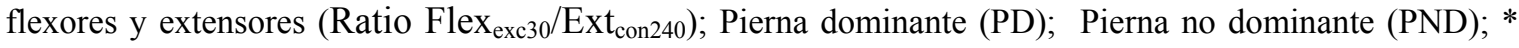
Significación $(\mathrm{p} \leq 0,05)$.

\section{Discusión}

La lesión de la musculatura isquiosural es una lesión común en el fútbol (Ekstrand y col., 2011), que además tiene una elevada tasa de reincidencia (Elliott y col., 2011; Orchard y Sweard, 2002; Petersen y Hölmich, 2005). Esto supone un coste deportivo y económico muy grande para deportistas y clubes profesionales (Benell y col., 1998). Diversos estudios indican que una de las causas de lesión isquiosural más relevantes es la existencia de desequilibrios entre la musculatura extensora y flexora de la rodilla (Croisier y col., 2002; Croisier, 2004; Jönhagen y col., 1994; Yeung y col., 2009), especialmente a causa de la debilidad de esta última. Por ello, se han desarrollado test de dinamometría isocinética de rodilla para evaluar el riesgo de lesión isquiosural a partir de parámetros como la ratio $\mathrm{Flex}_{\mathrm{exc} 30} / \mathrm{Ext}_{\mathrm{con} 240} \mathrm{o}$ el ángulo al que se alcanza el máximo momento de fuerza de la musculatura isquiosural (Brockett y col., 2004, Croisier y col., 2002). El objetivo principal de nuestro estudio fue analizar la relación de los valores de ratio $\mathrm{Flex}_{\mathrm{exc} 30} / \mathrm{Ext}_{\mathrm{con} 240} \mathrm{y}$ el ángulo de máximo momento de fuerza, con la incidencia de lesiones de isquiosurales sufridas por los jugadores a lo largo de una temporada, con el fin de proporcionar valores de referencia de este parámetro que permitieran la prevención de este tipo de lesiones. Atendiendo a los resultados obtenidos en nuestro estudio, valores bajos en la ratio $\mathrm{Flex}_{\text {exc30 }} / \mathrm{Ext}_{\mathrm{con} 240}$ parecen estar relacionados con un incremento en la probabilidad de sufrir lesión isquiosural en este tipo de deportistas. Resultados similares fueron obtenidos por Croisier y col. (2008), los cuales concluyeron que ratios inferiores a 0,89 pueden suponer una alta probabilidad de sufrir lesión. En nuestro estudio, los dos jugadores que presentaron ratios inferiores a 0,89 sufrieron lesión en la musculatura isquiosural durante la temporada. Por el contrario, el ángulo de máximo momento de fuerza no se mostró como un indicador útil para evaluar el riesgo de lesión isquiosural en este tipo de deportistas.

Estudios anteriores han utilizado varios valores diferentes de la ratio Flex $_{\text {exc30 }} /$ Ext $_{\text {con240 }}$ como indicadores de riesgo de lesión isquiosural en deportistas. Croisier y col. (2002) y Lehance, 
Moreno-Pérez, V.; Barbado-Murillo, D.; Juan-Recio, C.; Quesada-de-la-Gala, C.M.; Vera-García, F.J. (2013). Aplicación de la dinamometría isocinética para establecer perfiles de riesgo de lesión isquiosural en futbolistas profesionales. RICYDE. Rev. int. cienc. deporte. 34(9), 333-341. http://dx.doi.org/10.5232/ricyde2013.03403

Binet, Bury, y Croisier (2009) utilizaron una ratio de 0,80, mientras que Croisier y col. (2008) utilizaron una ratio de 0,89 . Ambas ratios indican una debilidad de la musculatura flexora de rodilla en relación a la musculatura extensora, si bien, según nuestros datos, 0,80 podría ser una ratio muy estricta, indicativa de un gran desequilibrio muscular, ya que sólo uno de los cinco futbolistas que se lesionaron durante la temporada obtuvo valores inferiores a esta ratio. Además, teniendo en cuenta que sólo uno de los cinco deportistas lesionados obtuvo valores superiores a 1,00 y que la mayor parte de deportistas que obtuvieron valores $\leq 1,00$ se lesionaron, valores inferiores o cercanos a 1,00, también deberían ser tenidos en cuenta como valores que incrementan la probabilidad de sufrir lesión isquiosural. Por tanto, un único límite dicotómico de 0,80 ó 0,89 parece demasiado sesgado a la hora de interpretar el riesgo de lesión. Quizá, se deberían establecer diferentes niveles de desequilibrio muscular y riesgo de lesión, como por ejemplo: elevado: ratios $<0,80$; moderado: ratios entre 0,80 y 0,90 ; leve: ratios entre 0,91 y 1,00 . No obstante, este planteamiento debe ser analizado en estudios con mayor número de futbolistas profesionales, ya que en nuestro trabajo, debido a la complejidad para acceder a esta población, la muestra no fue muy numerosa, lo que nos impidió realizar un análisis estadístico de los datos. De igual forma, para establecer con mayor precisión el riesgo de lesión

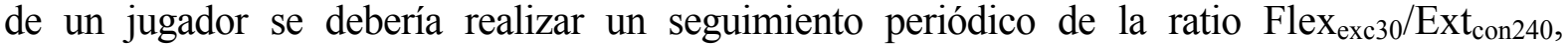
especialmente en aquellos jugadores con valores menores de 0,90 , ya que esta puede modificarse a lo largo de la temporada.

El ángulo donde la musculatura flexora de rodilla alcanzó su máximo momento de fuerza no fue un indicador válido del riesgo de lesión isquiosural. A diferencia de los trabajos de Brockett y col. (2004) y Proske y col. (2004), los máximos momentos de fuerza en las extremidades lesionadas no se obtuvieron en los menores grados de extensión. No obstante, es difícil hacer comparaciones entre los datos de nuestro estudio y los de estudios previos, ya que los test isocinéticos utilizados fueron diferentes. Así, por ejemplo, mientras en el estudio de Brockett y col. (2004) se analizó el ángulo donde la musculatura flexora de rodilla alcanzó su máximo momento de fuerza en contracción concéntrica (aceleración del segmento), en nuestro estudio el análisis se realizó en contracción excéntrica (frenado del segmento). Además, a diferencia de nuestro trabajo, en el estudio de Brockett y col. (2004) la muestra estaba compuesta por jugadores de fútbol australiano y atletas profesionales.

Cuando los futbolistas profesionales realizaron el test isocinético en pretemporada encontramos dos con antecedentes de lesión isquiosural, de los cuales, uno se lesionó durante la temporada. Debido a la elevada tasa de reincidencia, la existencia de antecedentes de lesión isquiosural es un factor importante para establecer perfiles de riesgo de lesión isquiosural (Orchard, 2001). Sin embargo, es interesante resaltar que el test isocinético realizado por el futbolista con antecedentes de lesión que se lesionó durante la temporada no presentó ratios Flex $_{\text {exc } 30} /$ Ext $_{\text {con240 }}$ o ángulos de máximo momento de fuerza que indicaran riesgo de lesión. Esto muestra la complejidad de este tipo de análisis y la gran cantidad de factores que interactúan y que deben ser interpretados conjuntamente para valorar el riesgo de lesión. Con el objeto de conformar una visión global del riesgo de lesión isquiosural de los futbolistas, futuros trabajos con dinamometría isocinética deberían incluir en su análisis todos aquellos factores que han sido asociados a la aparición de lesión en los isquiosurales: la existencia de lesiones previas (Orchard 2001), la edad del jugador (Gabbe y col., 2006), el déficit de flexibilidad muscular (Witvrouw y col., 2003), la baja estabilidad lumbo-pélvica (Sherry y Best, 2004), la fatiga (Greig y Siegler, 2009; Small y col., 2009), e incluso otros factores que no han sido estudiados hasta el momento, tales como la carga física a la que es sometido el futbolista a lo largo de una temporada, el sistema táctico del equipo y la posición del jugador dentro de dicho esquema táctico. 
Moreno-Pérez, V.; Barbado-Murillo, D.; Juan-Recio, C.; Quesada-de-la-Gala, C.M.; Vera-García, F.J. (2013). Aplicación de la dinamometría isocinética para establecer perfiles de riesgo de lesión isquiosural en futbolistas profesionales. RICYDE. Rev. int. cienc. deporte. 34(9), 333-341. http://dx.doi.org/10.5232/ricyde2013.03403

\section{Conclusiones}

Para concluir, podemos decir que la ratio $\mathrm{Flex}_{\mathrm{exc} 30} / \mathrm{Ext}_{\mathrm{con} 240}$ desarrollada por Croisier y col. $(2002,2008)$ a partir del test isocinético de rodilla puede ser un índice útil para determinar el riesgo o la probabilidad de lesión isquiosural en futbolistas profesionales. No obstante, es complejo establecer un único valor de ratio $(0,80$ ó 0,89$)$ que determine la existencia o no de riesgo de lesión en esta población. Según nuestros resultados, ratios inferiores o cercanas a 1,00, así como otros factores comentados en el párrafo anterior (antecedentes de lesión, edad del jugador, fatiga, etc.), deberían ser tenidos en cuenta conjuntamente para establecer perfiles de riesgo de lesión isquiosural en futbolistas profesionales. Por tanto, aunque la dinamometría isocinética es una técnica cara (Brughelli, Cronin, Mendiguchia, Kinsella, Nosaka, 2010), en el contexto económico de un club de fútbol profesional puede ser una inversión muy rentable, ya que un uso adecuado de la ratio $\mathrm{Flex}_{\mathrm{exc} 30} / \mathrm{Ext}_{\mathrm{con} 240}$, o de otros parámetros dinamométricos, complementado con el análisis de diversos factores de riesgo, podría reducir el elevado coste deportivo y económico del tiempo de inactividad de los jugadores lesionados.

\section{Agradecimientos}

Queremos agradecer la participación a todos los deportistas que prestaron su tiempo para participar en este estudio. El estudiante Casto Juan Recio ha podido participar en este estudio gracias a una beca pre-doctoral $(\mathrm{Val} \mathrm{i}+\mathrm{D})$ concedida por la Generalidad Valenciana.

\section{Referencias}

Bennell, K.; Wajswelner, H.; Lew, P.; Schall-Riaucour, A.; Leslie, S.; Plant, D., \& Cirone, J. (1998). Isokinetic strength testing does not predict hamstring injury in Australian Rule footballers. British Journal of Sports Medicine, 32(4), 309-314.

Brockett, C.L.; Morgan, D.L., \& Proske, U. (2004). Predicting hamstring strain injury in elite athletes. Medicine and Science in Sports and Exercise, 36(3), 379-387.

Brughelli, M.; Cronin, J.; Mendiguchia, J.; Kinsella, D., \& Nosaka, K. (2010). Contralateral leg deficits in kinetic and kinematic variables during running in Australian rules football players with previous hamstring injuries. Journal of Strength and Conditioning Research, 24(9), 2539-2544

Croisier, J.L.; Forthomme, B.; Namurois, M.H.; Vanderthommen, M., \& Crielaard, J.M. (2002). Hamstring muscle strain recurrence and strength performance disorders. American J ournal of Sports Medicine, 30(2), 199-203

Croisier, J.L. (2004). Factors associated with recurrent hamstring injuries. Sports Medicine, 34(10), 681-695

Croisier, J.L.; Ganteaume, S.; Binet, J.; Genty, M., \& Ferret, J.M. (2008). Strength imbalances and prevention of hamstring injury in professional soccer players: a prospective study. American J ournal of Sports Medicine, 36(8), 1469-1475

Dvorak, J., \& Junge, A. (2000). Football injuries and physical symptoms. American Journal of Sports Medicine, 28(5), S3-9.

Ekstrand, J.; Hägglund, M., \& Waldén, M. (2011). Epidemiology of muscle injuries in professional football (soccer). American J ournal of Sports Medicine, 39(6), 1226-1232.

Gabbe, B.J .; Bennell, K.L., \& Finch, C.F. (2006). Why are older Australian football players at greater risk of hamstring injury? J ournal of Science and Medicine in Sport, 9(4), 327333. 
Moreno-Pérez, V.; Barbado-Murillo, D.; J uan-Recio, C.; Quesada-de-la-Gala, C.M.; Vera-García, F.J. (2013). Aplicación de la dinamometría isocinética para establecer perfiles de riesgo de lesión isquiosural en futbolistas profesionales. RICYDE. Rev. int. cienc. deporte. 34(9), 333-341. http://dx.doi.org/10.5232/ricyde2013.03403

Greig, M., \& Siegler, J. C. (2009). Soccer-specific fatigue and eccentric hamstrings muscle strength. J ournal of Athletic Training, 44(2), 180-184.

Jönhagen, S.; Németh, G., \& Eriksson, E. (1994). Hamstring injuries in sprinters. The role of concentric and eccentric hamstring muscle strength and flexibility. American Journal of Sports Medicine, 22(2), 262-266

Lehance, C.; Binet, J.; Bury, T., \& Croisier, J. L. (2009). Muscular strength, functional performances and injury risk in professional and junior elite soccer players. Scandinavian J ournal of Medicine \& Science in Sports, 19(2), 243-251.

Orchard, J.W. (2001). Intrinsic and extrinsic risk factors for muscle strains in Australian football. American J ournal of Sports Medicine, 29(3), 300-303.

Petersen, J., \& Hölmich, P. (2005). Evidence based prevention of hamstring injuries in sport. British J ournal of Sports Medicine, 39(6), 319-323.

Proske, U.; Morgan, D.L.; Brockett, C.L., \& Percival, P. (2004). Identifying athletes at risk of hamstring strains and how to protect them. Clinical and Experimental Pharmacology and Physiology, 31(8), 546-550.

Sherry, M.A., \& Best, T.M. (2004). A comparison of 2 rehabilitation programs in the treatment of acute hamstring strains. The Journal of Orthopedic in Sports Physical Therapy, 34(3), 116-125.

Small, K.; McNaughton, L.; Greig, M., \& Lovell, R. (2009). Effect of timing of eccentric hamstring strengthening exercises during soccer training: implications for muscle fatigability. Journal of Strength and Conditioning Research, 23(4), 1077-1083.

Witvrouw, E.; Danneels, L.; Asselman, P.; D'Have, T., \& Cambier, D. (2003). Muscle flexibility as a risk factor for developing muscle injuries in male professional soccer players. A prospective study. American J ournal of Sports Medicine, 31(1), 41-46.

Woods, C.; Hawkins, R.D.; Maltby, S.; Hulse, M.; Thomas, A.; Hodson, A. (2004). The football association medical research programme: an audit of injuries in professional football- analysis of hamstring injuries. British Journal of Sports Medicine, 38(1), 3641.

Yeung, S.S.; Suen, A.M., \& Yeung, E.W. (2009). A prospective cohort study of hamstring injuries in competitive sprinters: preseason muscle imbalance as a possible risk factor. British J ournal of Sports Medicine, 43(8), 589-594. 